\title{
The perspectives of adults with aphasia and their team members regarding the importance of nine life areas for rehabilitation: A pilot investigation
}

\author{
Lauren K. Pettit, Kerstin M. Tönsing* and Shakila Dada \\ Centre for Augmentative and Alternative Communication \\ University of Pretoria
}

*Corresponding author:

e-mail: kerstin.tonsing@up.ac.za

Postal address: Centre for AAC, Communication Pathology Building, University of Pretoria, Hatfield, 0002 .

\section{Acknowledgements}

The authors would like to thank adults with aphasia, their significant others and speechlanguage pathologists who participated in this study.

\section{Funding and disclosure}

The financial assistance of the National Research Foundation (NRF) towards this research is hereby acknowledged. This work was also supported by a postgraduate student bursary from the University of Pretoria. Opinions expressed and conclusions arrived at, are those of the authors and cannot necessarily be attributed to the NRF or to the University of Pretoria. The authors have no non-financial relationships to disclose and they report no conflict of interests.

\section{Adherence to ethical standards}

The ethical board of the University of Pretoria approved the study. All ethical obligation towards participants and the scientific community were met. 


\begin{abstract}
Objectives: Client-centred rehabilitation implies that persons with aphasia and their significant others are actively involved in all decisions regarding rehabilitation, including the setting of rehabilitation priorities and goals. This study aimed to describe and compare the perspectives of adults with aphasia, their significant others and their speech-language pathologists (SLPs) regarding the importance of nine life areas for the rehabilitation of adults with aphasia.
\end{abstract}

Method: A total of 15 adults with expressive aphasia rated the importance of nine life areas using the Talking Mats ${ }^{\mathrm{TM}}$ framework. A questionnaire was used to obtain the ratings of 15 of their significant others and the 15 SLPs treating them.

Results: Most life areas were rated as important to work on in rehabilitation by most participants. The adults with aphasia rated the areas as important more frequently than their significant others and SLPs. All participants rated Communication as important. Statistically significant differences were noted for three of the nine life areas.

Discussion: The life areas which the participants were questioned about seem to provide a good starting point for rehabilitation teams to find common ground for collaborative goal setting. The Talking Mats ${ }^{\mathrm{TM}}$ approach allowed adults with aphasia to participate in the process. It can be a useful tool to promote client-centred rehabilitation for adults with expressive communication difficulties.

Keywords: Expressive aphasia; International Classification of Functioning, Disability and Health; Life area; Rehabilitation; Significant other; Speech-Language Pathologist; Talking Mats $^{\mathrm{TM}}$ framework 


\section{Introduction}

Adults with expressive aphasia post stroke present with reduced or limited verbal output and word retrieval difficulties. They typically experience difficulty communicating their basic daily needs and engaging in higher-level conversational tasks. ${ }^{1}$ As communication is an integral part of most human activities, the effects of aphasia are usually pervasive and manifest in reduced participation in various life areas., ${ }^{2,3,4}$ It has been suggested that rehabilitation programmes need to target broad participation-related goals to ensure meaningful outcomes..$^{5,6}$

The question arises as to what extent rehabilitation team members (including speechlanguage pathologists [SLPs], significant others and adults with aphasia themselves) share common views regarding the areas that are to be addressed in rehabilitation of adults with aphasia. While some SLPs embrace a holistic approach, ${ }^{7}$ others may continue to focus on contained discipline-specific goals. ${ }^{8,9}$ A functional, participation-focussed approach to communication intervention may be more aligned to the priorities of adults with aphasia and their significant others ${ }^{6}$.

Significant others are often directly affected by aphasia, ${ }^{5,6,8,10,11}$ and they play an important role in rehabilitation. ${ }^{12,13}$ Their needs and priorities for rehabilitation should therefore be considered in order to ascertain buy-in and appropriateness of therapeutic intervention to everyday contexts. At the same time, the perspectives of family members/friends and those of the person with aphasia do not always coincide when it comes to aspects concerning the life of the adult with aphasia. ${ }^{14,15}$ Significant others do not always perceive the disability resulting from aphasia in the same way as the adults themselves perceive it. ${ }^{16,17}$ While family voices are important, they should not replace those of the adults with aphasia themselves. 
Due to the communication challenges that adults with aphasia experience, obtaining their perspectives regarding their rehabilitation may be difficult. One method that has been used successfully in the past is the Talking Mats ${ }^{\text {TM }}$ Visual Framework. ${ }^{18,19,20,21}$ This framework consists of cards showing line drawings with written words that people with expressive difficulties can use to indicate choices and communicate their needs ${ }^{22,23}$ by placing the cards under specific headings. The Talking Mats ${ }^{\mathrm{TM}}$ Visual framework has been used in conjunction with aspects from the International Classification of Disability and Functioning (ICF) to enable people with communication difficulties to participate in goal setting. Bornman and Murphy ${ }^{24}$ adapted the terminology of the nine activities and participation dimensions from the ICF for easier understanding by people with communication difficulties. They represented each dimension or life area with Picture Communication Symbols $\left(\mathrm{PCS}^{\mathrm{TM}}\right)$ for the purpose of using this material within the Talking Mats ${ }^{\mathrm{TM}}$ framework. In a study conducted by Murphy and $\mathrm{Boa}^{22}$, people with long term communication difficulties (including those with aphasia) used this material to indicate whether they were 'managing' a life area and activities or tasks within that life area. Harty, Griesel and Van der Merwe ${ }^{25}$ also showed that individuals in an acute setting, who had experienced a head injury or stroke, were able to engage in a goal-setting activity using this material.

The aim of this study was to describe and compare the ratings given by adults with aphasia, their significant others and SLPs to the adapted activities and participation dimensions from the ICF to determine the importance of each of these nine broad life areas for the rehabilitation of the adult with aphasia. The ratings of adults were obtained using the Talking Mats ${ }^{\mathrm{TM}}$ Visual Framework. In this way, a method was piloted by which team perspectives regarding broad rehabilitation priorities could be determined and compared. 


\section{Methods}

\section{Participants}

The ethical board of the relevant higher education institution approved the study. Participants were recruited from six neurological rehabilitation facilities in two metropolitan areas in South Africa. A total of 15 adults with aphasia, their 15 significant others and the 15 SLPs rendering services to the adults with aphasia participated in the study. Inclusion criteria for the adults with aphasia were as follows: (a) mild, moderate or severe expressive aphasia (as determined by the oral language subtests of the Western Aphasia Battery [WAB], ${ }^{26}$ ) and medical history; (b) no or mild-moderate receptive language difficulties (determined by the comprehension subtests from the WAB, ${ }^{26}$ ); (c) premorbid English speaker; (d) receiving speech therapy at least every second week; (e) in the chronic stage of their rehabilitation (six months after the onset of stroke); (f) having a significant other who knew them well and who had some involvement in their rehabilitation; and (g) passing a screening task to determine whether visual, physical and cognitive abilities allowed them to participate in the Talking Mats ${ }^{\mathrm{TM}}$ activity (see screening task under 'Procedures').

The SLP was required to have at least one year of experience of neurological rehabilitation and needed to have treated the adult with aphasia for at least three months. Table 1 summarises some descriptive information in respect of the three groups of participants.

Of the adults with aphasia, four had mild anomia, six had moderate anomia and five had severe Broca's aphasia (as determined by the WAB). The mean Aphasia Quotient as determined by the WAB was 60.5 (SD 24.9), and the average time post onset was 19 months (SD: 8). Most received weekly speech-language therapy intervention (range: every second week to three times weekly). Seven of the adults presented with right hemiplegia, with a resulting change in handedness for six of the seven. The other eight adults had functional use 
of both hands. Eleven of the adults were mobile, two used a wheelchair and two were mobile with assistance of a device. All fifteen adults had a cerebral vascular accident in the left hemisphere of the brain. Of the significant others, seven were spouses of the adult with aphasia, three were family relations and five had another type of relationship (e.g., friend, fiancé). The significant others knew the person with aphasia for about 30 years on average (SD: 17.4, range 3-56 years). Ten of the significant others resided with the adult with aphasia, whereas five did not. Most (10) were in daily contact with the person with aphasia, with the others being in contact at least once a week. The SLPs had, on average, 6 years of experience working in neurorehabilitation, $(\mathrm{SD}=6.1$, range $1.4-20$ years $)$ and had been working with the adults with aphasia for an average of 15 months (SD: 6, range 4-24 months).

Table 1. Descriptive Information of Participants

Adults with $\quad$ Significant others $\quad$ SLPs

\begin{tabular}{|c|c|c|c|c|}
\hline & & aphasia & & \\
\hline Age & $\mathrm{M}$ & 55.3 & 50.0 & 31.5 \\
\hline & SD & 10.0 & 11.7 & 5.9 \\
\hline & Range & $38-71$ & $34-67$ & $26-42$ \\
\hline Gender ratio (M:F) & & $10: 5$ & $5: 10$ & $0: 15$ \\
\hline Highest level of & High school or lower & 6 & 8 & 0 \\
\hline & Diploma/graduate & 6 & 3 & 13 \\
\hline & degree & & & \\
\hline & Postgraduate degree & 3 & 4 & 2 \\
\hline
\end{tabular}

\section{Materials}

The WAB is designed to evaluate four main components of language function, while the Aphasia Quotient (AQ) indicates the severity level of aphasia. Expressive and receptive 
subtests from the $\mathrm{WAB}^{24}$ were used to determine eligibility and the severity of language difficulties.

For the screening task, nine Picture Communication Symbols ${ }^{\mathrm{TM}}(\mathrm{PCS}){ }^{27}$ printed on $4.5 \mathrm{~cm}$ x $4.5 \mathrm{~cm}$ cards were used. These cards depicted common objects such as food, water, a television, a tennis racquet, air, a dog, a house, clothing and a mobile phone. For the Talking Mats ${ }^{\mathrm{TM}}$ Interview, the material developed by Bornman and Murphy ${ }^{24}$ was used, comprising of nine $4.5 \mathrm{~cm} \mathrm{x} 4.5 \mathrm{~cm}$ topic cards that depicted nine life areas based on the activities and participation dimensions of the ICF. This material has been used in previous studies with people with long term and/or neurologically based communication difficulties ${ }^{22,25}$ to enable them to participate in goal setting. The life areas comprised of the following: Domestic Life, Relationships, Work and Education, Leisure, Self-care, Learning and Thinking, Coping, Communication and Mobility. The items were depicted using $\mathrm{PCS}^{27} . \mathrm{A}$ textured mat fitted with three category cards representing a three-point ordinal rating scale (Yes, Maybe, No) was used for both the screening task and the Talking Mats ${ }^{\mathrm{TM}}$ interviews. A questionnaire was provided for significant others and SLPs, requiring them to rate on a three-point ordinal rating scale (Yes, Maybe, No) each of the nine life areas in terms of whether or not they saw this area as important for the adult with aphasia to work on in rehabilitation.

\section{Procedures}

Consent and screening. Informed consent was obtained from all participants. Additional measures were taken to ensure that adults with aphasia had the opportunity to grant informed consent without being coerced and with full knowledge of the study ${ }^{21}$.

Participants then completed a biographical questionnaire. The significant other or researcher (first author) assisted the adult with aphasia to complete the questionnaire. The 
researcher subsequently administered the subtests from the $\mathrm{WAB}^{26}$ after which the screening task was conducted with the adult with aphasia. This screening task was based on previously published studies ${ }^{18,25}$ and aimed to ensure that participants had the necessary visual and physical ability to take part in the Talking Mats ${ }^{\mathrm{TM}}$ interview and were able to comprehend the task. The adult with aphasia was asked to determine the importance of each of nine items (each depicted on a card) for survival, and was required to respond by placing each card under one of the following headings: Yes, Maybe or No. Each heading was supported by a pictorial image. To qualify for inclusion, participants were required to place the three cards depicting food, water and air under the heading Yes. All adults with aphasia passed this screening.

Data collection. Significant others and SLPs completed questionnaires to rate the importance of each life area for the rehabilitation of the adult with aphasia. A page with life area descriptions was attached to the questionnaires to provide examples of items under each area. All the significant others and two of the SLPs completed their questionnaires at the same location (but in separate rooms) and at the same time as the researcher was conducting the Talking Mats ${ }^{\mathrm{TM}}$ interview with the adult with aphasia. The 13 SLPs who could not be present at this meeting were contacted a few days before the scheduled meeting and the consent forms, biographical questionnaires and life area questionnaires (along with written instructions) were emailed to them for completion.

Adults with aphasia completed their rating of the nine life areas during an individual Talking Mats ${ }^{\mathrm{TM}}$ interview with the researcher. The researcher presented the adult with each of the nine cards depicting life areas. She also provided a written and verbal description and examples of each life area. She then asked the question "Is it important for you to work on improving [name of life area] in your life?" The adult with aphasia was required to respond by placing the card under the appropriate heading on the mat. For example, the researcher 
would show the adult the Self-care card and ask, 'Is it important for you to work on improving Self-care in your life?' and the adult could answer by placing the card on the mat under Yes, Maybe or No. After the nine cards representing the life areas had been placed on the mat, the adults with aphasia were asked to check that they were satisfied with their choices. A photograph of the completed mat was taken. The Talking Mats ${ }^{\mathrm{TM}}$ interviews were video-recorded for analysis, for the sake of both procedural and inter-observer reliability.

Data Analysis. The first author captured the responses provided by each participant from the digital photographs of the completed Talking Mats ${ }^{\mathrm{TM}}$ interviews as well as from the completed questionnaires in MS Excel spread sheets. The frequency of Yes, Maybe and No responses for each life area and within each group (adults with aphasia, significant others and SLPs) was calculated. Fisher's exact test was used to determine whether there was a statistically significant difference between the groups in terms of the areas that were rated as important.

\section{Procedural integrity and data reliability}

To establish procedural integrity, the Talking Mats ${ }^{\mathrm{TM}}$ interviews were video-recorded. A postgraduate student in psychology watched $30 \%$ of the video recordings (randomly selected) and completed a checklist to determine to what extent the researcher had followed the proposed procedures. The percentage of steps adhered to was calculated to determine procedural integrity. Overall, the researcher adhered to $87 \%$ of the steps, indicating good procedural consistency for the Talking Mats ${ }^{\mathrm{TM}}$ interview. The same postgraduate student also checked the reliability of the data capturing by independently capturing $30 \%$ of the data (randomly selected) in MS Excel spread sheets. Percentage agreement was 100\%, indicating good reliability. 


\section{Results}

\section{Ratings of adults with aphasia}

The percentages of Yes, Maybe and No ratings of the nine life areas provided by adults with aphasia are presented in Figure 1.

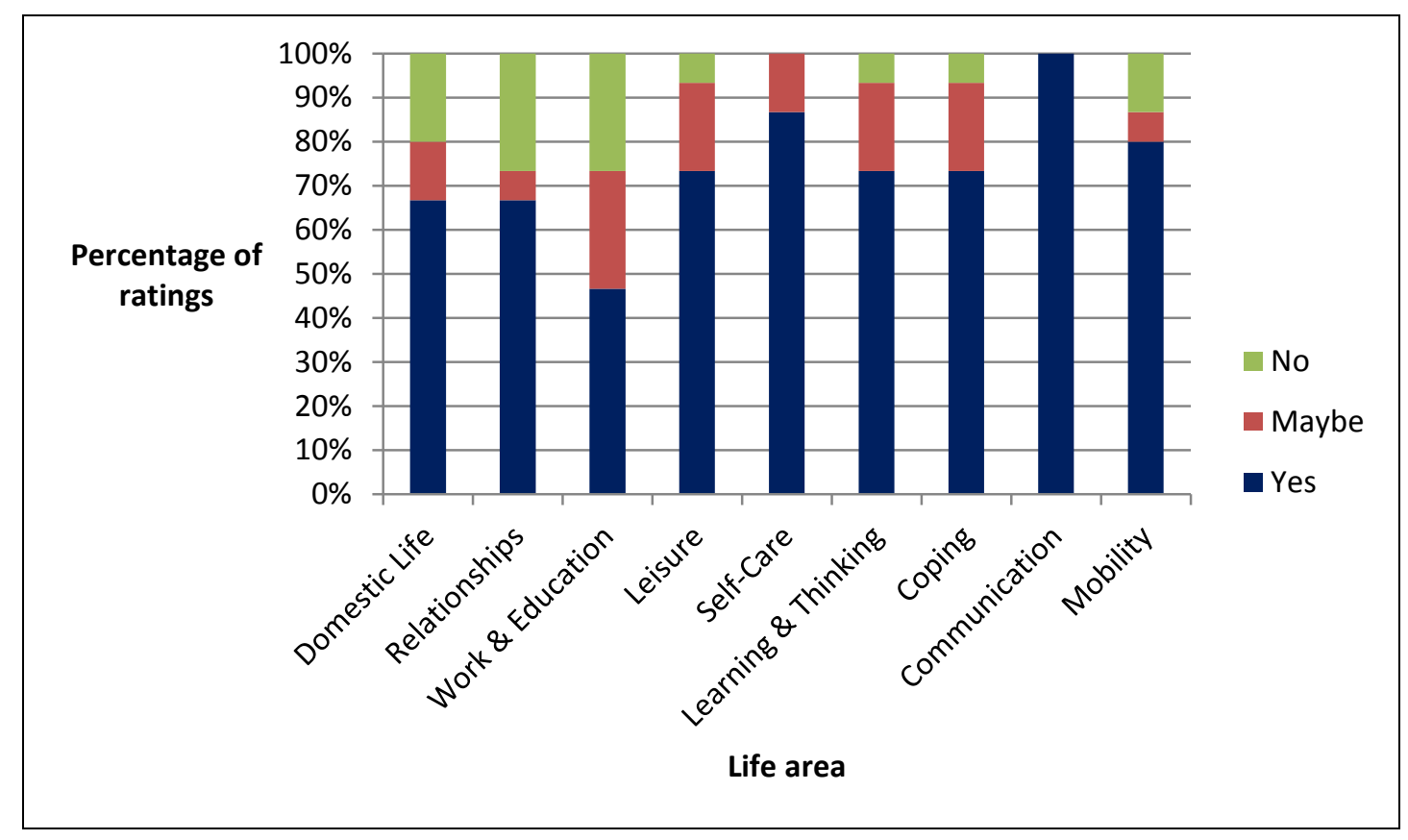

Figure 1. Percentages of Yes, Maybe and No ratings for the nine life areas provided by adults with aphasia.

All areas received more Yes ratings than Maybe or No ratings. The highest percentages of Yes ratings within this study were awarded to the following areas: Communication (100\%), Selfcare (86.7\%), Mobility (80\%) and Leisure, Learning and Thinking and Coping (73.3\%). The adults with aphasia identified all these areas as important areas for rehabilitation.

\section{Ratings by significant others}

The percentages of Yes, Maybe and No ratings of the nine life areas provided by significant others are presented in Figure 2. 




Figure 2. Percentages of Yes, Maybe and No ratings for the nine life areas provided by significant others for adults with aphasia.

While six of the nine areas received more Yes than No ratings, significant others clearly gave more Maybe and No ratings than adults with aphasia did. The areas with the highest percentages of Yes ratings were Communication (86.67\%), and Learning and Thinking as well as Coping (53.3\% each). The highest No ratings were given to Leisure (53.3\%), Mobility (46.7\%), as well as Work and Education and Self-care (40\% each).

\section{Ratings by SLPs}

The percentages of Yes, Maybe and No ratings of the nine life areas provided by SLPs are presented in Figure 3. 


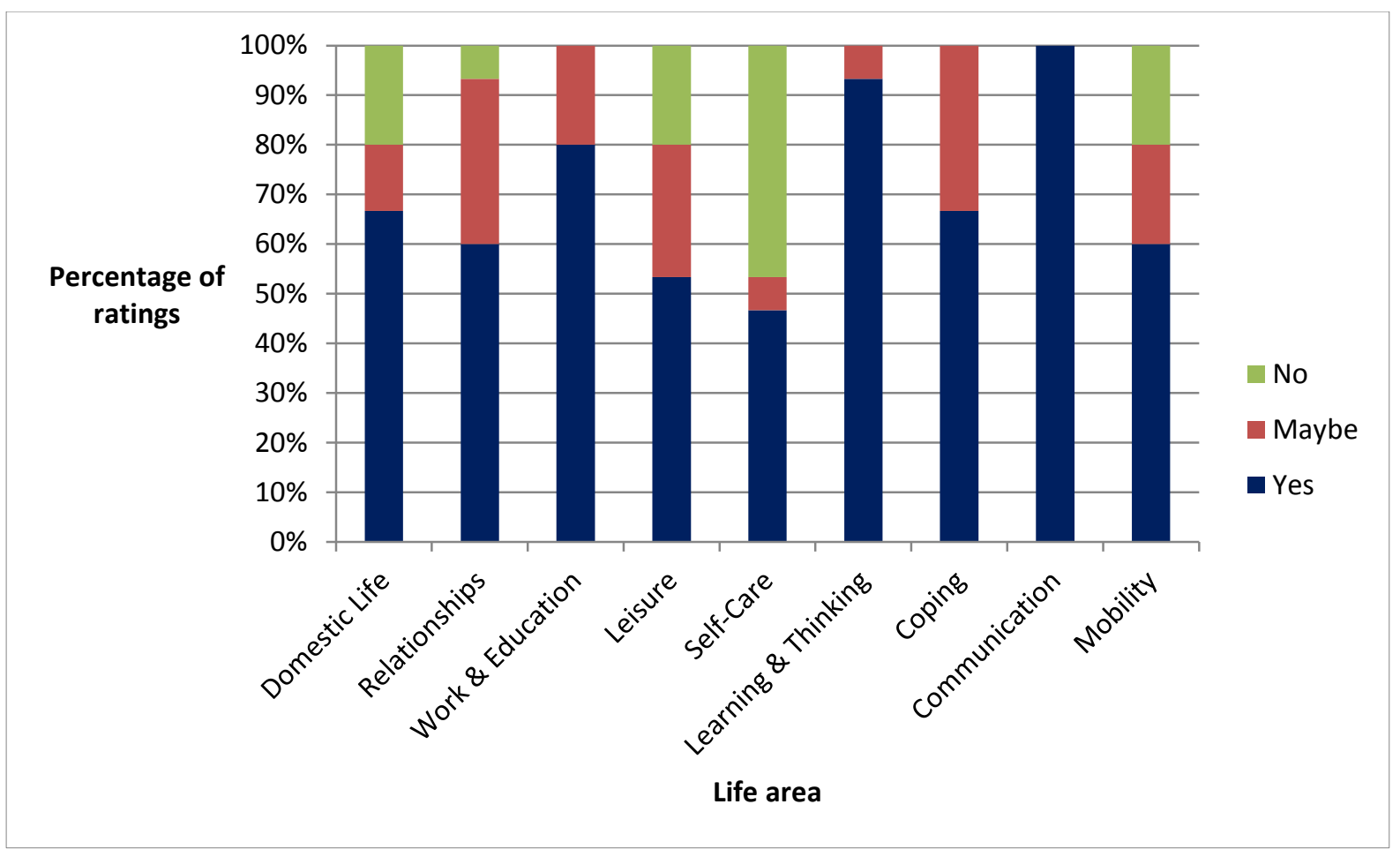

Figure 3. Percentages of Yes, Maybe and No ratings for the nine life areas provided by SLPs for adults with aphasia.

SLPs rated eight of the nine with more Yes than No ratings. They gave slightly more Maybe and No ratings than the adults with aphasia did. The highest percentage of Yes ratings were awarded for Communication (100\%), Learning and Thinking (93.3\%) and Work and Education (80\%). The area that received the highest No rating was Self-care (46.7\%).

\section{Comparison of the ratings of life areas}

Fisher's exact test was used to obtain the p-values and effect sizes. According to these values, statistically significant differences were found in the response patterns for adults with aphasia, significant others and SLPs for only three life areas, namely Work and Education (p $=0.0233$, medium effect size), Leisure $(\mathrm{p}=0.0258$, medium effect size $)$, and Self-care $(\mathrm{p}=$ 0.0143, medium effect size). Table 2 presents the overall ratings of the areas awarded by the adults with aphasia, their significant others and SLPs. 
Table 2. The Percentages of Yes, Maybe and No Ratings for each Life Area across the Groups and Corresponding Fisher Exact Test p-Values

\begin{tabular}{|c|c|c|c|c|c|c|c|c|c|c|c|}
\hline \multirow[b]{3}{*}{ Life area } & \multicolumn{9}{|c|}{ Percentage of responses } & \multirow[b]{3}{*}{ p-value } & \multirow{3}{*}{ Effect size } \\
\hline & \multicolumn{3}{|c|}{ AA } & \multicolumn{3}{|c|}{ SLP } & \multicolumn{3}{|c|}{$\mathrm{SO}$} & & \\
\hline & $\overline{\text { Yes }}$ & Maybe & No & Yes & Maybe & No & Yes & Maybe & No & & \\
\hline Domestic life & 67.67 & 13.33 & 20.0 & 66.67 & 13.33 & 20.0 & 46.67 & 20.0 & 33.33 & 0.8314 & 0.19 \\
\hline Relationships & 66.67 & 6.67 & 26.67 & 60.0 & 33.33 & 6.67 & 46.67 & 33.33 & 37.50 & 0.2581 & 0.39 \\
\hline Work \& Education & 46.67 & 26.67 & 26.67 & 80.0 & 20.0 & 0.0 & 26.67 & 33.33 & 40.0 & $0.0233^{*}$ & 0.48 \\
\hline Leisure & 73.33 & 20.0 & 6.67 & 53.33 & 26.67 & 20.0 & 20.0 & 26.67 & 53.33 & $0.0258 *$ & 0.49 \\
\hline Self-care & 86.67 & 13.33 & 0.0 & 46.67 & 6.67 & 46.6 & 46.67 & 13.33 & 40.0 & $0.0143 *$ & 0.46 \\
\hline & & & & & & 7 & & & & & \\
\hline Coping & 73.33 & 20.0 & 6.67 & 66.67 & 33.33 & 0.0 & 53.33 & 33.33 & 13.33 & 0.6067 & 0.26 \\
\hline Communication & 100.0 & 0.0 & 0.0 & 100.0 & 0.0 & 0.0 & 86.67 & 6.67 & 6.67 & 0.3182 & 0.30 \\
\hline Mobility & 80.0 & 6.67 & 13.33 & 60.0 & 20.0 & 20.0 & 46.67 & 6.67 & 46.67 & 0.1892 & 0.38 \\
\hline
\end{tabular}

Note $. \mathrm{AA}=$ adults with aphasia $\mathrm{SO}=$ significant others. Effect size: $0.1=$ small, $0.3=$ medium, $0.5=$ large $* p<0.05$

\section{Discussion}

All three participant groups selected more Yes than Maybe or No ratings, indicating that all three groups regarded most of the areas as important to address in rehabilitation for most of the adults with aphasia. Harty et al. ${ }^{25}$ found similar results when adults in an acute setting, post stroke or head injury, and their service providers rated the importance of these nine life areas for rehabilitation, while Worrall et al. ${ }^{5}$ found that most of the goals which adults with aphasia expressed in semi-structured interviews aligned with the activities and participation dimensions of the ICF. In the current study, persons with aphasia as well as their families and SLPs were clearly aware of the effect of aphasia on a variety of life areas, such as relationships, leisure and work aspects. Statistically significant differences between the ratings of the three groups were only found in three of the nine areas, suggesting that team members generally had similar views about the broad rehabilitation priorities. This is 
considered a positive finding, as agreement among team members regarding such priorities can lay the foundation for an integrated, client-driven approach towards intervention. ${ }^{7,9,28}$

In this study, the significant others overall gave less Yes ratings across the life areas than adults with aphasia, suggesting that they prioritised these areas for rehabilitation less frequently than adults with aphasia themselves. This is in contrast to studies by Cruice et al. ${ }^{16}$ and Hesketh et al. ${ }^{17}$, which showed that significant others perceived the disability to be worse than adults with aphasia perceived it. There may be various reasons for this - the significant others in this study may have had a more positive view of the functioning of the adults with aphasia than the adults themselves had. They may also have perceived that certain areas (e.g. Work and Education) were not relevant to the lives of the adults with aphasia.

The fact that the SLPs in the current study selected many of the life areas as important for adults with aphasia to work on, suggests that they were able to focus on participation goals rather than only on discipline-specific goals, as was previously suggested in some studies $^{8,9}$. Similar results were found by Harty et al. ${ }^{25}$, as well as Brown et al. ${ }^{10}$, who found that SLPs were well aware that adults with aphasia tend to select goals related to participation and life activities rather than to focus on discrete, discipline-specific goals. These results articulate well with the life participation approach to aphasia (LPAA) as advocated by the LPAA group $^{6}$ in 2001, which called for communication intervention approaches for persons with aphasia and significant others that focussed on 'reengagement in life' (p. 279), and noted that life activities targeted in SLP intervention did not need to pertinently fall into the realm of communication.

Regarding similarities and differences between the ratings that the three groups assigned to specific areas, Communication clearly received the highest Yes ratings by all three groups. Due to the communication difficulties experienced by adults with aphasia, it is not surprising that this area was rated as important by all the adults with aphasia, most of the 
significant others and all SLPs. Many of the significant others in this study were family members (spouse or child) or close friends. They spent a great deal of time with the adult with aphasia and would have experienced their daily communicative difficulties and stresses. $^{29}$

Three areas (Work and Education, Leisure and Self-care) were rated significantly differently by the three groups. Most SLPs (80\%) felt that Work and Education was an important area to focus on, while less than half (47\%) of the adults with aphasia and only about a quarter of the significant others $(27 \%)$ rated this area as important. Self-care received a high number of Yes ratings from adults with aphasia, whereas less than half of the significant others and SLPs rated this area as important. Regarding Leisure, many adults with aphasia (73\%) felt that this was an important area to work on, whereas only $20 \%$ of significant others gave a Yes rating for this area. The small sample size and the fact that a 3point scale (rather than a more nuanced rating scale) was used demand caution in interpreting these observed differences, and reasons for these differences also remain speculative. It is possible that these life areas may be perceived as not falling directly within the scope of practice of SLPs. Self-Care, for example (focused on activities of daily living such as the ability to dress, wash and groom oneself) is traditionally seen as the responsibility of the occupational therapist ${ }^{30}$ rather than that of the SLP ${ }^{31}$, and SLPs in the current study may not have been aware of self-care needs. On the other hand, SLPs seemed more aware of mobility needs, a domain traditionally falling within the physical therapist's scope of practice, ${ }^{32}$ although the SLPs in this study rated this domain as important less often than adults with aphasia. Certainly the disagreements observed highlight areas that would need further exploration regarding the reasons for different viewpoints and methods of reaching consensus in terms of rehabilitation priorities. 
Clinically, these life areas can provide the 'common language' for team members to engage in dialogue and identify problem areas related to the daily life functioning of people with expressive aphasia. By simplifying some of the labels of the activities and participation dimensions of the ICF and pairing these labels with pictures and the interactive Talking Mats ${ }^{\mathrm{TM}}$ interview procedure, adults with expressive aphasia (who often have difficulty participating in the selection of rehabilitation priorities) were able to express their own views. This may be a first step in assisting the adult with aphasia to advocate for themselves and to exercise their right to identify the activities and participation opportunities which they would like to access, and to set rehabilitation priorities based on their choice. ${ }^{3,6}$ While the overlap in priorities amongst the three groups as found in this study is encouraging, the presence of some significant differences underlines the importance of the voice of adults with aphasia themselves. This ensures truly client-centred rehabilitation that underscores the principles of human rights and a focus on competence rather than deficits. ${ }^{6}$ At the same time, significant others are also profoundly affected by the presence of aphasia and are often intimately involved in assisting the person with aphasia to participate in daily life activities. When differences in priorities become apparent through a process as the one used in this study, the question should not be whose voice counts, but rather how the fact that each voice was heard can provide a platform for negotiating priorities amongst team members in a way that each party's autonomy is respected and maintained.

For SLPs, a focus on life participation can ensure that functional outcomes explicitly remain the ultimate goal and the ultimate measure of effectiveness of intervention, ${ }^{3,6}$ and that environmental factors that may act as barriers (e.g., lack of mobility aids preventing a person from accessing communication environments) be addressed where possible. ${ }^{3}$ Since the life areas addressed in this study are not discipline-specific, future studies may also include other professionals such as physical and occupational therapists to obtain a more comprehensive 
picture of team perspectives as a basis for collaboration. Regrettably, many adults with chronic aphasia do not receive intervention from a variety of professionals due to limited public and private medical aid budgets. ${ }^{6}$

Results from this study are limited by the small sample size. Since only 15 adults with expressive aphasia, their respective significant others and SLPs participated, the findings of this study have limited generalisability. Also, reasons for the ratings assigned were not explored in great detail. Future studies may attempt to explore the reasons for specific ratings by team members in a more systematic fashion. Identified life areas could also be explored in greater detail by rating specific tasks and activities relating to this life area. By aligning these more directly to the particular second, third and fourth level domains mentioned under the activities and participation dimensions of the ICF, the individual codes appearing under the chapters in the ICF manual could be explored. In this way, specific aspects of concern within a specific life area can be highlighted, and a more comprehensive picture of similarities and differences in perspectives may be obtained. Future studies may also explore the usability of the life areas as an intervention tool to build team consensus through facilitating dialogue among team members about the similarities and differences in their ratings.

\section{Conclusion}

In summary, based on the relatively high percentage of Yes ratings given by all three groups of participants, the current study confirms that the life areas which the participants were questioned about represent aspects that should be addressed in rehabilitation. Overall, similarities found in the ratings suggest that the team members in the current study prioritised many of the same areas for rehabilitation, and that these teams would be able to find common ground to identify rehabilitation goals agreed upon and supported by everyone in the team. However, it was also found that the perspectives of some areas were not always shared 
among team members. These discrepancies highlight the need to explore how perspectives can be communicated and priorities can be negotiated.

\section{Funding and disclosure}

The financial assistance of the National Research Foundation (NRF) towards this research is hereby acknowledged. This work was also supported by a postgraduate student bursary from the University of Pretoria. Opinions expressed and conclusions arrived at, are those of the authors and cannot necessarily be attributed to the NRF or to the University of Pretoria. The authors have no non-financial relationships to disclose and they report no conflict of interests.

\section{REFERENCES}

1. Lasker, J, Hux, K, Garrett, KL, Moncrief, EM, \& Eischeid, TJ. Variations on the written choice communication strategy for individuals with severe aphasia. Augment Altern Commun. 1997;13:108-116.

2. Simmons-Mackie, N, \& Kagan, A. Application of the ICF in aphasia. Semin Speech Lang. 2007;28(4):244-53.

3. Threats, T. Access for persons with neurogenic communication disorders: influences of personal an environmental factors of the ICF. Aphasiology, 2007;21(1):67-80. doi: $10.1080 / 02687030600798303$

4. Worrall, L, McCooey, R, Davidson, B, Larkins, B \& Hickson, L. The validity of functional assessments of communication and the Activity/Participation components of the ICIDH-2: do they reflect what really happens in real-life? J Comm Disord. 2001;35:107-137. 
5. Worrall, L, Sherratt, S, Rogers, P, Howe, T, Hersh, D, Ferguson, A, \& Davidson, B. What people with aphasia want: Their goals according to the ICF. Aphasiology. 2011;25(3):309-322.

6. Chapey, R, Duchan, JF, Elman, RJ, Garcia, LJ, Kagan, A, Lyon, JG, Simmons-Mackie, N. Life-participation approach to aphasia: a statement of values for the future. In: Chapey, R, ed. Language Intervention Strategies in Aphasia and Related Neurogenic Communication Disorders. $4^{\text {th }}$ ed. Philadelphia, PA: Lippincott, Williams \& Wilkins; 2001:235-245.

7. Brown, K, Worrall, LE, Davidson, B, \& Howe, T. Living successfully with aphasia: A qualitative meta-analysis of the perspectives of individuals with aphasia, family members, and speech-language pathologists. Int Journal Speech Lang Pathol. 2012;14(2):141-155. doi: 10.3109/17549507.2011.632026

8. Hersh, D, Worrall, L, Howe, T, Sherratt, S, \& Davidson, B. SMARTER goal setting in aphasia rehabilitation. Aphasiology. 2012;26(2):220-233. doi:

$10.1080 / 102687038.2011 .640392$

9. Sherratt, S, Worrall, L, Pearson, C, Howe, T, Hersh, D, \& Davidson, B. "Well it has to be language-related": Speech-language pathologists' goals for people with aphasia and their families. Int J Speech Lang Path. 2012;13(4):317-328. doi:

$10.3109 / 17549507.2011 .584632$

10. Brown, K, Worrall, L, Davidson, B \& Howe, T. Living successfully with aphasia: Family members share their views. Top Stroke Rehabil. 2011;18(5):536-548.

11. Worrall, L, Brown, K, Cruice, M, Davidson, B, Hersh, D, Howe, T, \& Sherratt, S. The evidence for life-coaching approach to aphasia. Aphasiology. 2010;24(4):497-514. doi: $10.1080 / 02687030802698152$ 
12. Threats, T. The ICF framework and third party disability: application to spouses of persons with aphasia. Top Stroke Rehabil. 2010;6:451-457. doi: http://0dx.doi.org.innopac.up.ac.za/10.1310/tsr1706-451

13. Paul, NA \& Sanders, GF. Strategies used by communication partners of aphasic speakers. Act, Adapt Aging. 2009;33(3):161-178.

14. Engell, B, Hutter, BO, Willmes, K \& Huber, W. Quality of life in aphasia: Validation of a pictorial self-rating procedure. Aphasiology. 2003;17(4):383-396.

15. Hilari, K, Owen, S, \& Farrelly, SJ. Proxy and self-report agreement on the Stroke and Aphasia Quality of Life Scale- 39. J Neurol Neurosurg Psychiatry. 2007;78(10):10721075. doi: 10.1161/01.STR.0000081987.46660.ED

16. Cruice, M, Worrall, L, Hickson, L, \& Murison, R. Measuring quality of life: Comparing family members' and friends' ratings with those of their aphasic partners. Aphasiology, 2005;19(1):111-129.

17. Hesketh, A, Long, A, \& Bowen, A. Agreement on outcome: Speaker, carer, and therapist perspectives on functional communication after stroke. Aphasiology. 2011;25(3):291-308. doi: 10.1080/02687038.2010.507818

18. Beringer, A, Tönsing, K, \& Bornman, J. The self-determined and partner-predicted topic preferences of adults with aphasia. Aphasiology. 2012;27(2):1-25. doi: $10.1080 / 02687038.2012 .744809$

19. Murphy, J. Enabling people with aphasia to discuss quality of life. Br J Therap Rehab. 2000;7(11):454-458. doi: 0743-4618/97/1302-0092

20. Murphy, J, Gray, CM., \& Cox, S. Using 'Talking Mats' to help people with dementia to communicate. https://www.jrf.org.uk/report/using-talking-mats-help-peopledementia-communicate. Accessed January 24, 2016 
21. Murphy, J. \& Cameron, L. The effectiveness of Talking Mats ${ }^{\mathrm{TM}}$ with people with intellectual disability. Br J Learn Disabil. 2008 36(4):232-241.

22. Murphy, J, \& Boa, S. Using the WHO-ICF with Talking Mats to enable adults with long-term communication difficulties to participate in goal setting. Augment Altern Commun. 2012;28(1):52-60. doi: 10.3109/07454618.2011.653828

23. Murphy, J, Tester, S, Hubbard, G, Downs, M, \& MacDonald, C. Enabling frail older people with a communication difficulty to express their views: the use of Talking Mats $^{\mathrm{TM}}$ as an interview tool. Health Soc Care Community. 2005;13(2):95-107.

24. Bornman, J, \& Murphy, J. Using the ICF in goal setting: Clinical application using Talking Mats ${ }^{\text {TM }}$. Disabil Rehabil Assist Technol. 2006;1(3):145-154. doi: $10.1080 / 17483100612331392745$

25. Harty, M, Griesel, M, \& Van der Merwe, A. The ICF as a common language for rehabilitation goal-setting: comparing client and professional priorities. Health Qual Life Outcomes. 2011;9(87):1-9.

26. Kertesz, A. Western Aphasia Battery. New York: Grune \& Stratton: 1982.

27. Mayer-Johnson. PCS ${ }^{\mathrm{TM}}$ Symbols - Picture Communication Symbols. 2015. http://www.mayer-johnson.com/category/symbols-and-photos. Accessed January 24, 2016.

28. Leach, E, Cornwell, P, Fleming, J, \& Haines, T. Patient-centred goal setting on the subacute rehabilitation setting. Disabil Rehabil. 2010;32(2):159-172. doi: $10.3109 / 09638280903036605$.

29. Johansson, MB., Carlsson, M, Ostberg, P, \& Sonnander, K. Communication changes and SLP services according to significant others of persons with aphasia. Aphasiology. 2012;26(8):1005-1028. doi: 10.1080/02687038.2012.671927\ 
30. American Occupational Therapy Association. Scope of practice. 2014.

http://www.aota.org/-/media/corporate/files/aboutaota/officialdocs/position/scope-ofpractice-edited-2014.pdf. . Accessed May 5, 2014.

31. American Speech-Language-Hearing Association. Scope of practice in speechlanguage pathology. 2007. http://www.asha.org/uploadedFiles/SP2007-00283.pdf. Accessed January 11, 2016.

32. American Physical Therapy Association. The physic al therapist scope of practice. 2015. http://www.apta.org/ScopeOfPractice/\#print. Accessed January 11, 2015. 